\title{
¿Un solo ventilador para varios pacientes?
}

\section{Single ventilator for multiple patients?}

\section{José L. Sandoval-Gutiérrez*}

Instituto Nacional de Enfermedades Respiratorias "Ismael Cosío Villegas", Subdirección de Servicios Auxiliares de Diagnóstico, Ciudad de México, México

El doctor Castañón González et al. publicó un interesante artículo acerca de la posibilidad terapéutica de ventilación mecánica simultánea: ${ }^{1}$ describen un trabajo experimental con el cual identificaron que no había diferencias estadísticas significativas en los diferentes parámetros ventilatorios.

Este escenario fue pensado debido al déficit de ventiladores ante la pandemia de COVID-19. En contra de la recomendación al respecto, algunos especialistas consideraron que esta opción podría ser necesaria, si bien constituía una medida extrema. Hasta el momento desconocemos si algún centro la ha implementado, probablemente no por las connotaciones éticas y bioéticas que conlleva.

Conectar a un solo ventilador a dos o más pacientes con síndrome de dificultad respiratoria, diferentes mecánicas respiratorias y estados basales de gravedad, complicaría el manejo y seguimiento de los casos. Aun cuando los circuitos disponen de filtros, el control de las infecciones no sería el óptimo. Si la intención es tanatológica, se pudiera considerar dicho fin, pero, por supuesto, no sería una medida terapéutica.

Si bien la sabiduría popular dice "donde comen dos, comen tres", aún no puede señalarse que "donde se ventila uno, se ventilan dos".

\section{Bibliografía}

1. Castañón-González JA, Camacho-Juárez S, Gorordo-Delsol LA, Garduño-López J, Pérez-Nieto $O$, Amezcua-Gutiérrez MA, et al. Ventilación mecánica simultánea con un solo ventilador a varios pacientes. Gac Med Mex. 2020;156:250-253.
Gac Med Mex. 2020;156:367

Disponible en PubMed 